\title{
High-density recording in photopolymer-based holographic three-dimensional disks
}

\author{
Allen Pu and Demetri Psaltis
}

\begin{abstract}
The performance specifications of a holographic three-dimensional disk system are experimentally characterized. A surface density of $10 \mathrm{bits} / \mu \mathrm{m}^{2}$ is experimentally demonstrated with a $100-\mu \mathrm{m}$-thick photopolymer as the recording medium.

Key words: Holographic storage, disk, photopolymer. ㅅ 1996 Optical Society of America
\end{abstract}

\section{Introduction}

We examine the use of Dupont's photopolymer as the holographic recording material for the implementation of a holographic three-dimensional (3-D) disk. ${ }^{1,2}$ A schematic diagram of such a system is shown in Fig. 1. The holographic disk is a photopolymer film laminated on a glass substrate in the shape of a disk. The disk is rotated by a mechanical drive, as in a conventional disk system, while the optical head is considerably more complex than the head of a regular compact-disk (CD) player. For a read-write system, the optical head consists of a spatial light modulator (SLM), optics, a mechanism for multiplexing holograms, and a two-dimensional (2-D) detector array. Any location on the disk can be addressed by the combined mechanical motions of the disk rotation and the translation of the optical head radially. Multiple holograms are overlapped at each location by the use of hologram-multiplexing methods such as angle,,$^{3,4}$ wavelength, ${ }^{5,6}$ phase-code, ${ }^{7,8}$ fractal, ${ }^{9,10}$ peristrophic, ${ }^{11}$ and shift ${ }^{12}$ multiplexing. The particular implementation we describe in this paper utilizes a combination of angle and peristrophic multiplexing. However, most of our conclusions apply to a disk implemented with any multiplexing method.

Data are recorded in the form of 2-D pages presented on the SLM. An optical system transfers the SLM information onto the disk. A hologram is formed by the recording of the interference between

The authors are with the Department of Electrical Engineering, California Institute of Technology, 116-81 Caltech, 1201 East California Boulevard, Pasadena, California 91125.

Received 27 July 1995; revised manuscript received 10 October 1995.

0003-6935/96/142389-10\$10.00/0

(1) 1996 Optical Society of America this signal beam and a plane-wave reference beam. The recorded page is read out by the illumination of the hologram, with the plane-wave reference used for recording. The reconstruction is imaged onto the CCD. If the angle of the reconstructing beam is rotated in the plane defined by the wave vector of the recording reference and the central ray of the signal beam, then the hologram becomes Bragg mismatched because of the finite thickness of the medium, and the reconstruction disappears almost completely. Additional holograms can be recorded and independently retrieved with such rotated references. If the readout plane wave is rotated around the surface normal, as shown in Fig. 1, then, for relatively thin media, the hologram remains Bragg matched and a reconstruction is produced. However, the rotation of the readout beam induces a proportional change in the angle of the reconstructed beam. If this angular change is larger than the angular bandwidth of the recorded hologram, it can be blocked by the insertion of an aperture in the system. A new hologram can then be recorded at this new position of the reference beam. The process is repeated indefinitely until the reference is rotated by $360^{\circ}$. The number of holograms that can be superimposed at one location by the combination of these two multiplexing techniques (angle and peristrophic) is determined by the thickness of the medium and the bandwidth of the signal beam.

Holograms in the holographic 3-D disk can be recorded in the image, Fourier, or Fresnel planes. Selection of one method over another is based primarily on the available optics, SLM's, detector arrays, quality of the resulting holograms, complexity of the setup, and surface density. To maximize the surface density of a holographic 3-D disk, it is necessary to pack as many bits into as small a surface as possible. Therefore, for image-plane holograms, 


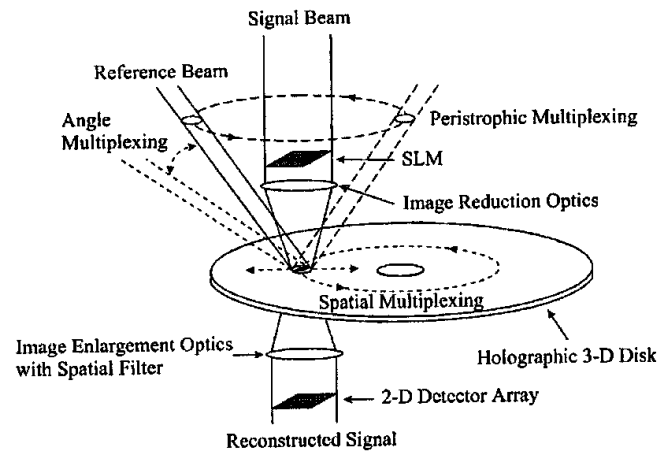

Fig. 1. Schematic diagram of a holographic 3-D disk system that uses a combination of angle and peristrophic multiplexing. SLM, spatial light modulator.

each pixel (bit) must be as small as possible. On the other hand, to achieve high surface density with Fourier-plane holograms, it is better to have large pixels so that the Fourier transform is small (the Fourier transform of a square pixel produces a spot size in one dimension that is equal to $2 \lambda F / \delta$, where $\lambda$ is the wavelength, $F$ is focal length, and $\delta$ is the pixel size). Although there are expensive stepper lenses that can produce large images with pixels less than a micrometer in size, it is generally easier to obtain lenses that resolve large pixels. Furthermore, image-plane holography generally requires more lenses than Fourier-plane holography does for relaying the image to the recording material and then to the readout detector array. One disadvantage of recording Fourier-transformed holograms is that the intensity of the signal beam is usually nonuniform. Consequently, the quality of the reconstructed hologram is poor because of the strong dc present during recording. One way to get around this problem is to use a pixel-to-pixel matched phase diffuser right after the SLM. This way, the signal beam will be approximately uniformly distributed in the Fourier plane while the size of the recording spot does not increase. Another method to fix this problem is to record the hologram slightly off of the exact Fourier plane (Fresnel plane). In the Fresnel plane, the intensity is more evenly distributed at the cost of a slightly larger spot size. We used Fresnel-plane recording in our experiments.

The most important performance characteristics of holographic 3-D disks are the surface density, the recording characteristics (rate and linearity), the readout rate, and the signal-to-noise ratio (SNR) of the reconstructions. We assembled an experimental apparatus with which we evaluated these performance characteristics for a holographic disk implemented with 100- $\mu$ m-thick Dupont photopolymer. A diagram of the experimental setup is shown in Fig. 2. A glass plate of a random binary bit pattern was used as the input SLM. The center-to-center spacing of the pixels was $45 \mu \mathrm{m}$, and the fill factor was $100 \%$. Nikon $F / 1.4,3.9$-cm-aperture camera lenses were used for imaging (the glass mask plate was pressed up against the Nikon lens to ensure that all

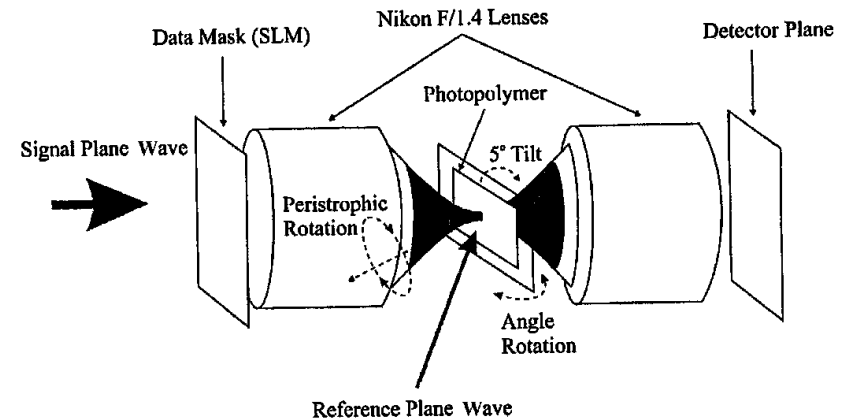

Fig. 2. Diagram of the experimental setup in which Dupont's 100- $\mu$ m-thick photopolymer was used.

the pixels within the lens aperture were captured). A total of 590,000 pixels fit in the apertures of the two Nikon lenses, and a sharp image of the entire field was obtained at the CCD plane. The holograms were recorded with a plane reference beam approximately $0.5 \mathrm{~mm}$ past the Fourier-transform plane. At that position, the diameter of the signal beam was $1.5 \mathrm{~mm}$ and its spatial uniformity was much better than that at the exact Fourier plane. For simplicity, peristrophic multiplexing was achieved by rotation of the recording material, instead of the reference beam, around the surface normal. Angle multiplexing was combined with peristrophic multiplexing to increase the number of pages stored at each location.

In the remainder of the paper, we report a sequence of experimental results from this apparatus that gives us a good estimate for the performance we can expect from a polymer-based holographic disk. Specifically, in Section 2 we derive the surface density realizable with the 100- $\mu$ m-thick film. In Section 3 we describe the recording characteristics of the polymer that yield the linearity and the recording rate of the system. In Section 4 we measure the diffraction efficiency of the holograms and thus determine the achievable readout rate, and in Section 5 we report our measurements of the fidelity of the reconstructions and show that a density of at least $10 \mathrm{bits} / \mathrm{\mu m}^{2}$ can be achieved with a low probability of error. Finally, we summarize the results and conclude in Section 6.

\section{Surface Density}

The surface density of current CD memories is approximately $1 \mathrm{bit} / \mu^{2}$ and is limited primarily by the size of the illuminating spot. The new generation of optical CD's that are scheduled to appear in the near future will likely have a storage density of ${ }^{13} 5$ bits $/ \mu \mathrm{m}^{2}$ per layer. Dual-layer and double-sided systems are expected to have an equivalent storage density in excess of $10 \mathrm{bits} / \mu^{2}$ For holographic memories to be competitive, the density of holographic disks must be higher than the projected density of conventional media by a comfortable margin. This concern makes storage density the primary goal in the design of a holographic disk. The storage density of holographic disks was ana- 
lyzed by Li and Psaltis, ${ }^{2}$ who showed that, for the optimum thickness of the recording medium $(L=1.6$ $\mathrm{cm}$ ), we can obtain a density of $110 \mathrm{bits} / \mathrm{\mu m}^{2}$ by using angle multiplexing. This gives more than an order of magnitude of margin over the projected $5 \mathrm{bits} / \mathrm{\mu m}^{2}$ of conventional CD technology. The method we present in this paper can achieve higher densities than those predicted in Ref. 2 for relatively thin recording media, such as the photopolymer.

To a first approximation, the density of a holographic disk is given by

$$
D_{3 \mathrm{D}}=M D_{2 \mathrm{D}}
$$

where $D_{3 \mathrm{D}}$ and $D_{2 \mathrm{D}}$ are the surface densities achievable with 3-D and 2-D mediums, respectively, and $M$ is the number of holograms multiplexed. The density predicted by Eq. (1) is a theoretical upper limit that can be difficult to achieve in practice. For example, Eq. (1) holds only if the resolution of the conventional CD lens and the resolution of the lens in the signal arm of the system in Fig. 2 are the same. This is possible but difficult because the 3-D memory must maintain the same resolution over a large field, whereas the CD lens needs to produce only a single spot at the optical axis of the lens. The recording medium's dynamic range can also limit the storage density, as the diffraction efficiency falls off as $1 / M^{2}$. Finally, some noise sources, such as cross talk, increase as $M$ gets larger. In a practical system, the storage density is maximized when the optical design is optimized while the SNR of the reconstructed holograms is monitored.

The maximum number of holograms that can be angularly multiplexed at each location is given by

$$
M_{\text {angle }}=\frac{\Theta}{\Delta \theta}=\frac{\Theta L \sin \left(\theta_{r}+\theta_{s}\right)}{\lambda \cos \left(\theta_{s}\right)},
$$

where $\Theta$ is the range of angles that can pass through the aperture of the optical system in the reference arm, $\Delta \theta$ is the angular selectivity, $L$ is the thickness of the hologram, $\lambda$ is the wavelength of the laser, $\theta_{r}$ $\left(\theta_{s}\right)$ is the angle between the reference (signal) beam and the disk surface normal (in this paper, all parameters are referenced to inside the recording material). We can obtain an expression for the 3-D storage density by substituting Eq. (2) into Eq. (1). If we assume that $D_{2 \mathrm{D}}$ is independent of $L$ (reasonable for relatively thin media) then we obtain $D_{3 \mathrm{D}} \sim$ $L$. In Fig. 3 we plot the surface density as a function of $L$, which we calculated by using the analysis of Ref. 2 and also Eqs. (1) and (2) from this paper. We see that the approximate analysis yields a density estimate that is very close to the estimate of the detailed analysis for thicknesses of up to $100 \mu \mathrm{m}$. Therefore we use Eq. (1) to calculate the density of a disk that is implemented with the $100-\mu m$-thick polymer.

For our experimental setup, $L=100 \mu \mathrm{m}, \lambda=349$ $\mathrm{nm}\left(532 \mathrm{~nm}\right.$ outside), $\theta_{r}=22^{\circ}$, and $\theta_{s}=16^{\circ}$, which gives $\Delta \theta$ a value of $0.31^{\circ}$ to the first null of the sinc

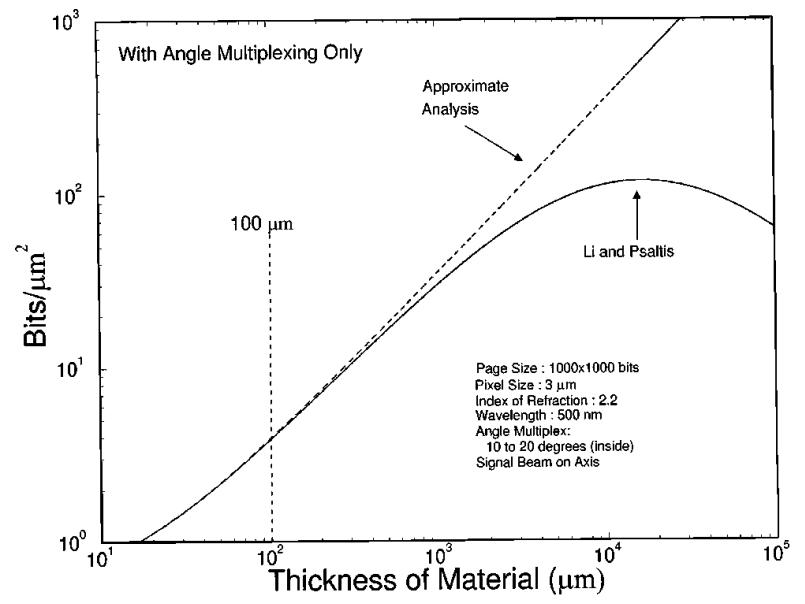

Fig. 3. Holographic 3-D disk surface density as a function of the recording-material thickness. The solid curve is the prediction from Ref. 2, and the dotted curve is the approximate analysis used in this paper.

function (the index of refraction for Dupont's photopolymer is 1.525). However, Eq. (2) is valid only for plane-wave holograms. The signal-beam spread inside was nearly $26^{\circ}$ because of the large numerical aperture of the $F / 1.4$ Nikon lenses. When three holograms were angularly multiplexed, the SNR's measured were 4 and 3.2 for angular separations of $3.3^{\circ}$ and $1.3^{\circ}$, respectively, whereas the SNR measured for a single recorded hologram was 4.5. Therefore we selected to separate the holograms angularly by $1.6^{\circ}\left(2.5^{\circ}\right.$ outside angle $)$ to strike a balance between density and cross-talk noise. Consequently, with $\Delta \theta=1.6^{\circ}$ and a geometrically limited $\Theta$ of approximately $14^{\circ}$, up to eight holograms can be angularly multiplexed at a given location. Because the photopolymer has enough dynamic range to record more than eight holograms, we introduce peristrophic multiplexing to increase the storage density.

As shown in Fig. 1, peristrophic multiplexing is achieved when the reference beam is rotated around the film's surface normal after each hologram is recorded (or when the film is rotated around the reference beam). The rotation causes the reconstruction to exit at a different direction where it can be filtered, allowing a new hologram to be stored and retrieved without cross talk. The change in peristrophic angle required for moving the reconstruction off the detector is independent of $L$. For Fourier-plane holograms, the amount of rotation required for shifting the reconstructed image off of the detector array is $^{11}$

$$
\Delta \psi_{\text {Fourier }}=\frac{d / F}{\sin \theta_{r}+\sin \theta_{s}},
$$

where $d$ is the size of the reconstructed image in the detector plane and $F$ is the focal length of the lens used. For image-plane holograms, the amount of rotation required for filtering out the undesired 
reconstruction in the Fourier plane of the system with a spatial filter is

$$
\Delta \psi_{\text {Image }}=\frac{(2 \lambda) / \delta}{\sin \theta_{s}+\sin \theta_{r}},
$$

where $1 / \delta$ is the highest spatial frequency in the image. Peristrophic multiplexing in either the image or the Fourier planes gives essentially no cross talk between the recorded holograms; this is because the undesired holograms are blocked and do not interfere with the desired hologram.

When $\theta_{r}=\theta_{s}$, it is possible to multiplex peristrophic holograms in the range between 0 and $\pi$ before degeneracy occurs. If the signal is perpendicular to the film surface $\left(\theta_{s}=0\right)$ and $\theta_{r}$ is large enough, then it is possible to multiplex peristrophic holograms from 0 to $2 \pi$. For example, if the goal is to store many holograms in the same location without regard to density, we can choose $d=1 \mathrm{~cm}, F=50 \mathrm{~cm}, \delta=50$ $\mu \mathrm{m}, L=100 \mu \mathrm{m}, \lambda=349 \mathrm{~nm}, \theta_{r}=30^{\circ}$, and $\theta_{s}=0^{\circ}$. Then $\Delta \psi_{\text {Fourier }}=2.3^{\circ}$, and $\Delta \psi_{\text {Image }}=1.6^{\circ}$. Therefore up to 225 holograms can be recorded by the use of peristrophic multiplexing in the image plane. On the other hand, if the surface density is important, then we might chose $d=3.9 \mathrm{~cm}, F=5.5 \mathrm{~cm}, \delta=1$ $\mu \mathrm{m}, L=100 \mu \mathrm{m}, \lambda=349 \mathrm{~nm}, \theta_{r}=22^{\circ}$, and $\theta_{s}=16^{\circ}$ (the exact setup used in our experiment, except for $\delta$ ). Then $\Delta \psi_{\text {Fourier }}=63^{\circ}$ and $\Delta \psi_{\text {Image }}=61.5^{\circ}$. In this case, only up to two peristrophic holograms can be recorded. In our setup, the recording material was tilted an additional $5^{\circ}$ in the plane perpendicular to the plane formed by the reference and the signal beams to enhance the effects of peristrophic rotation on shifting the spectrum off of the $\kappa$ sphere. Experimentally, we found that in this tilted configuration, $45^{\circ}$ in rotation was sufficient to completely Bragg mismatch and shift the undesired hologram off of the detector array. Therefore up to four peristrophic holograms can be multiplexed from 0 to $\pi$ with this configuration. Sets of eight angularly multiplexed holograms were recorded at each of the four different peristrophic positions. We achieved angle multiplexing by rotating the film in the plane formed by the signal and the reference beams instead of by changing the reference-beam angle. Using this method, we stored a total of $M=8 \times 4=$ 32 holograms at a single location. A window from one of the 32 reconstructions is shown in Fig. 4.

Having determined $M$, we now need to calculate $D_{2 \mathrm{D}}$ to find the density of the 3-D disk. We compute $D_{2 \mathrm{D}}$ by dividing the total number of SLM pixels that are holographically recorded $(590,000)$ by the area occupied by the signal beam on the medium $(\pi \times 0.75$ $\mathrm{mm} \times 0.75 \mathrm{~mm})$. Using this definition, we obtain $D_{2 \mathrm{D}}=0.334 \mathrm{bits} / \mathrm{um}^{2}$. Because 32 holograms are superimposed in the same location, the overall surface density is $32 \times 0.334=10.68 \mathrm{bits} / \mu \mathrm{m}^{2}$. We can further improve the density of the current system by using lower $f$-number lenses (higher resolution and density per page), reducing the angular separation

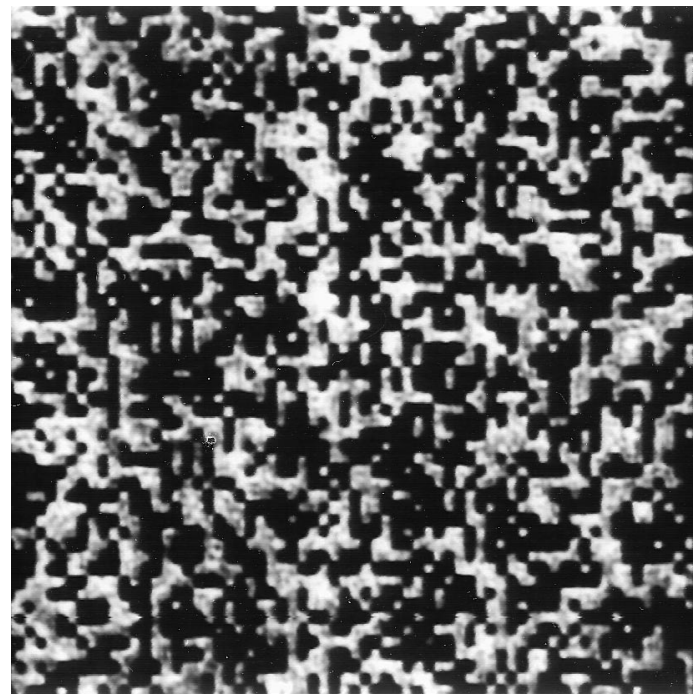

Fig. 4. Reconstructed image from one of the 32 holograms stored.

between holograms, and increasing the range of angles over which angle holograms can be recorded. The density can of course be increased by a large factor (see Fig. 3) if a thicker recording medium is used. The small thickness is the most serious shortcoming of the Dupont polymer for the 3-D disk application.

\section{Recording Rate and Linearity}

The rate at which data can be recorded on the holographic disk, without taking into account the overhead of multiplexing, is

$$
R_{\text {in }}=N_{p} / \tau_{\text {in }}
$$

where $N_{p}$ is the number of pixels per page and $\tau_{\text {in }}$ is the time required for recording one hologram.

The recording time $\tau_{\text {in }}$ is determined by the sensitivity of the recording medium and the desired diffraction efficiency per hologram. For Dupont's 100 - $\mu$ m-thick photopolymer (HRF-150), the diffraction efficiency as a function of exposure energy is shown in Fig. 5. We obtained Fig. 5 by integrating the diffraction efficiencies of 50 plane-wave peristrophic holograms, each exposed to $7 \mathrm{~mJ} / \mathrm{cm}^{2}$ with $\lambda=$ $320 \mathrm{~nm}$ (488 nm outside) and $\theta_{r}=\theta_{s}=19.2^{\circ}$. This particular recording material is fairly insensitive until it is exposed by $\sim 35 \mathrm{~mJ} / \mathrm{cm}^{2}$. It then exhibits a quasi-linear recording behavior and flattens out as the material nears saturation at $\sim 325 \mathrm{~mJ} / \mathrm{cm}^{2}$. This means that the holograms recorded later in the sequence require a longer exposure time for achieving the same diffraction efficiency as the earlier holograms. We use a recording schedule that compensates for the loss of grating strength as the material saturates, thereby using all the available dynamic range of the material.

We derive the recording schedule by taking the square root of Fig. 5 to obtain the hologram grating strength as a function of exposure energy; we then model this curve by fitting it to a sixth-order polyno- 


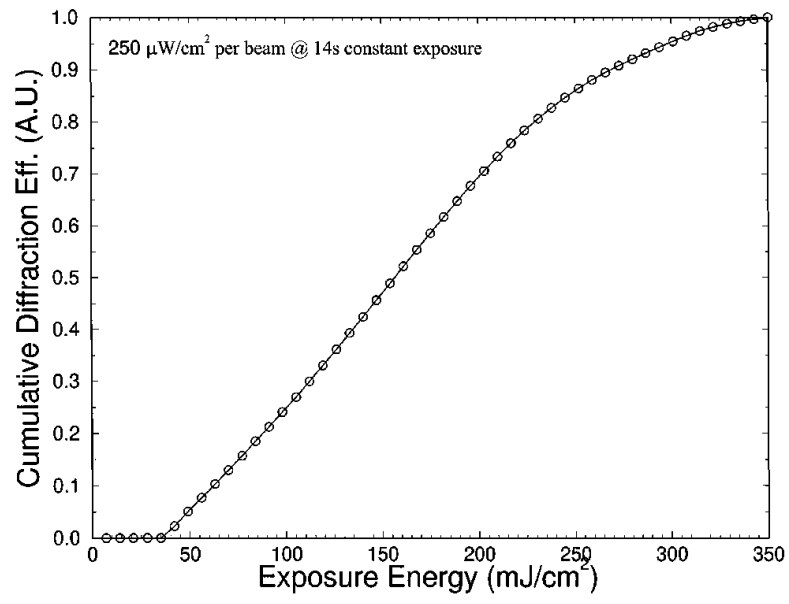

Fig. 5. Diffraction efficiency as a function of exposure energy for Dupont's HRF-150-100 photopolymer.

mial, $F(E)$. When the derivative of the polynomial is taken with respect to exposure energy, the local grating growth rate, $f(E)=F^{\prime}(E)$ (in units of square centimeters per joule), is obtained as a function of exposure energy. Given $f(E)$, the strength of each hologram to be multiplexed can be predetermined if the exposure energy is allocated appropriately; specifically, equal-strength holograms can be recorded if the entire dynamic range of the photopolymer is allocated equally among the holograms. The desired exposure for the $n$th hologram to achieve equal diffraction efficiencies for all the holograms is

$$
t_{n}=\frac{A_{\mathrm{sat}}}{M f\left(E_{n-1}\right) I},
$$

where $t_{n}$ is the exposure time for the $n$th hologram, $A_{\text {sat }}$ is the saturation grating strength, $M$ is the number of holograms, $E_{n-1}$ is the cumulative exposure energy up to the $n-1$ th hologram, and $I$ is the exposure intensity. We preexpose the material with $35-\mathrm{mJ} / \mathrm{cm}^{2}$ worth of energy $\left(E_{0}=35 \mathrm{~mJ} / \mathrm{cm}^{2}\right)$ to make it sensitive before recording any holograms.

An exposure schedule for 50 holograms was calculated with Eq. (6), and Fig. 6 shows the result. Note that the exposure time per hologram increases as the film becomes more saturated. The diffraction efficiencies of the 50 holograms that were recorded with the schedule shown in Fig. 6 were more uniform than when equal exposure times were used, but were still not equal. This is because the scheduled exposure time changes the overall exposure the film receives before the $n$th exposure, compared with the equal exposure schedule with which Fig. 5 was calculated. We take this run-time effect ${ }^{14}$ into account by iterating the above procedure of integrating the square root of the diffracted power from the 50 newly recorded holograms, fitting the new curve to the polynomial, and then generating a new schedule by using Eq. (6). An interesting by-product of this recording procedure is that the saturation grating strength $A_{\text {sat }}$ increases by a few percent for the

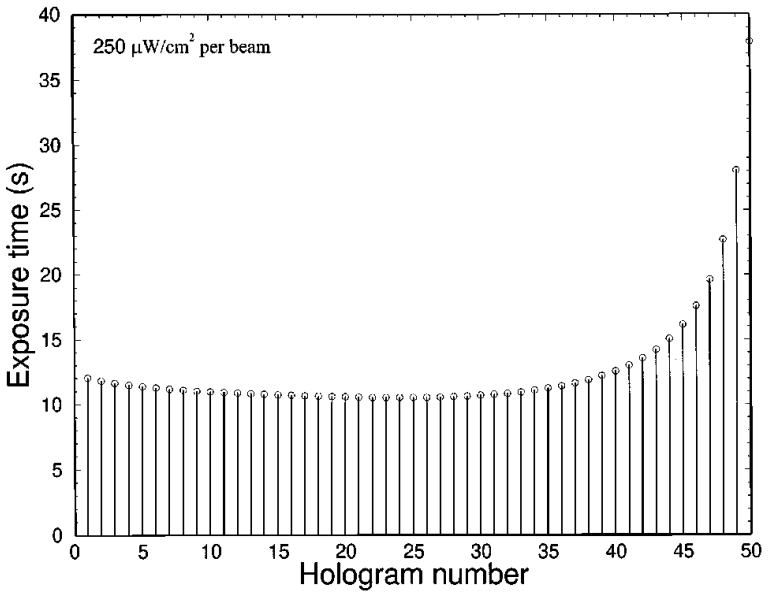

Fig. 6. Scheduled exposure time as a function of the hologram number for 50 holograms.

scheduled recording. Figure 7 shows the diffraction efficiency of the 50 holograms that were recorded with two iterations of the recording schedule. From Fig. 7 it can be seen that the diffraction efficiency is uniformly distributed among the 50 holograms. The above iterative procedure can be repeated until the desired uniformity is reached.

When the recording schedule is used, the recording time is different for each page of data, and therefore the recording rate also changes. If a buffer is used to store all the holograms that are to be recorded at one location, then the appropriate measure is the average recording rate:

$$
\bar{R}_{\text {in }}=\frac{M N_{P}}{\sum_{n=1}^{M} \tau_{\text {in }}(n)}=\frac{M^{2} N_{P} I}{A_{\text {sat }} \sum_{n=1}^{M} \frac{1}{f\left(E_{n-1}\right)}} .
$$

From the parameters of our experimental setup we predict, from Eq. (7), a recording rate of 130 $\mathrm{kbits} / \mathrm{s}$. In the high-density experiment, however, we were not able to use the recording-schedule

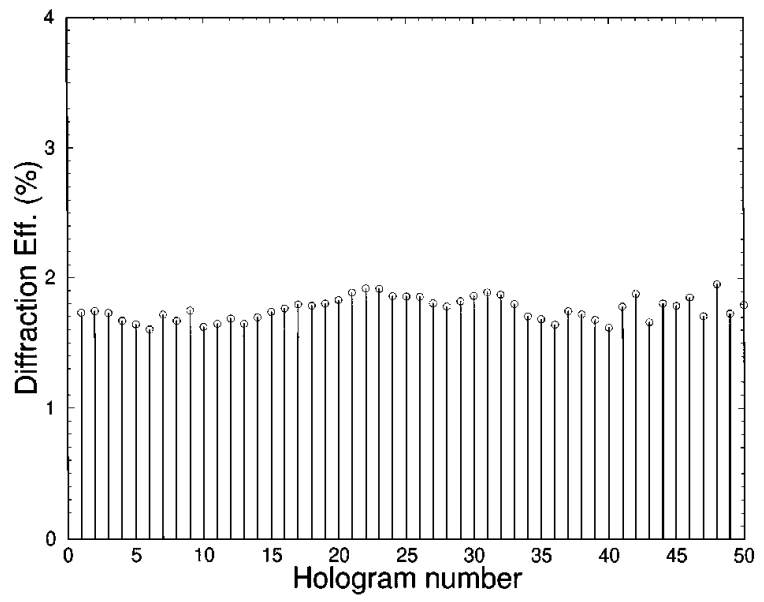

Fig. 7. Diffraction efficiency of 50 holograms recorded with the second iteration of the recording schedule. 
procedure as described above. This is because the intensity of the reference beam inside the material varied by $\sim 10 \%$ as its angle was changed by $13^{\circ}$ because of the Fresnel reflections from the film surface. This variation in intensity leads to additional nonuniformity in the recorded holograms. These variations can in principle be eliminated by antireflection coating or compensation of the reference-beam amplitude. They can also be removed by continued iterations of our basic exposure-schedule procedure if a higher-order polynomial is used to model the curve that describes the dependence of the cumulative grating strength on the exposure. Instead, we were able to obtain the recording schedule for equalizing the 32 holograms simply through trial and error.

In selecting the exposure time we are faced with the trade-off between diffraction efficiency and quality of the reconstructions. Clearly, if the entire dynamic range of the recording material is used, then the diffraction efficiency of each of the equalized holograms is maximized. On the other hand, the quality of the reconstructions degrades when the entire dynamic range is used. This degradation occurred when we attempted to record high-density data in the Fresnel plane. We believe that this effect is due to the index modulation in the recording area from the multiple exposures (or, equivalently, interpixel gratings) that distort the images traveling through the medium. To minimize this effect, we restricted the recording to only $\sim 30 \%$ of the dynamic range of the medium.

A recording rate of $0.7 \mathrm{Mbits} / \mathrm{s}$ was experimentally demonstrated in the high-density setup. This was done when 590,000 pixel holograms were recorded in an average recording time of $840 \mathrm{~ms}$ per hologram. The total incident intensity was $2 \mathrm{~mW} / \mathrm{cm}^{2}$, and the diffraction efficiency per hologram was $\sim 0.35 \%$. From Eq. (7) and our experimental observations with plane-wave holograms, we know that the recording time for achieving the same diffraction efficiency is approximately inversely proportional to the incident intensity for intensities greater than $2 \mathrm{~mW} / \mathrm{cm}^{2}$. For intensities less than $2 \mathrm{~mW} / \mathrm{cm}^{2}$, the diffraction efficiency drops as the recording intensity is increased, whereas the total exposure energy remains the same because of the diffusion-limited process inherent in this material. ${ }^{15}$ Therefore, if the total incident intensity is increased from $2 \mathrm{~mW} / \mathrm{cm}^{2}$ to $128 \mathrm{~mW} / \mathrm{cm}^{2}$, then the average recording time per hologram drops to $13 \mathrm{~ms}$, and the recording rate becomes $45 \mathrm{Mbits} / \mathrm{s}$. Note that the recording rate we obtained experimentally $(0.7 \mathrm{Mbits} / \mathrm{s})$ is substantially faster than the rate predicted by Eq. (7) 130 $\mathrm{kbits} / \mathrm{s}$ ). This is because we used only a small, linear portion of the material's dynamic range in the experiment.

\section{Readout Rate}

One of the limiting factors for the readout rate is the time $\tau$ it takes to accumulate a sufficient number of photons per CCD pixel so that the reconstructions can be reliably detected above the noise floor. We can estimate $\tau$ as

$$
\tau=\frac{P h c N_{p}}{\eta I_{\text {inc }} \lambda_{\text {outside }}},
$$

where $P$ is the minimum number of photons per pixel, $h$ is Planck's constant, $c$ is the speed of light, $N_{p}$ is the total number of pixels, $\eta$ is the diffraction efficiency, $I_{\text {inc }}$ is the incident power of the readout beam, and $\lambda_{\text {outside }}$ is the wavelength outside of the material. We use a generous choice of $P=1000$ photons for the calculation. The rest of the parameters in Eq. (8), except for the hologram diffraction efficiency $\eta$, have already been determined in our experimental setup. If we multiplex $M$ holograms in a linear, saturable material by using the full dynamic range of the material, then the amplitude of each recorded hologram becomes ${ }^{16} R * S \propto A_{\text {sat }} / M$. Because the diffraction efficiency is proportional to the grating strength squared, the diffraction efficiency can be written as

$$
\eta=\left(\frac{M / \#}{M}\right)^{2},
$$

where the quantity $M$ /\# characterizes the efficiency of the material in the recording geometry that it is being used. As we have already determined that $M=32$, we can determine $\eta$ by measuring the $M / \#$. We recorded, in the HRF-150-38 (product number of the $38-\mu \mathrm{m}$ Dupont polymer), sets of $10,25,50,75$, and 90 plane-wave holograms by using peristrophic multiplexing and the recording schedule described above. The same procedure was performed for the HRF-150-100 (the 100- $\mu$ m-thick polymer) with sets of $25,50,75$, and 90 plane-wave holograms. The resulting diffraction efficiencies are plotted in Fig. 8 as functions of the number of holograms. We can see that the experimental data agree well with the

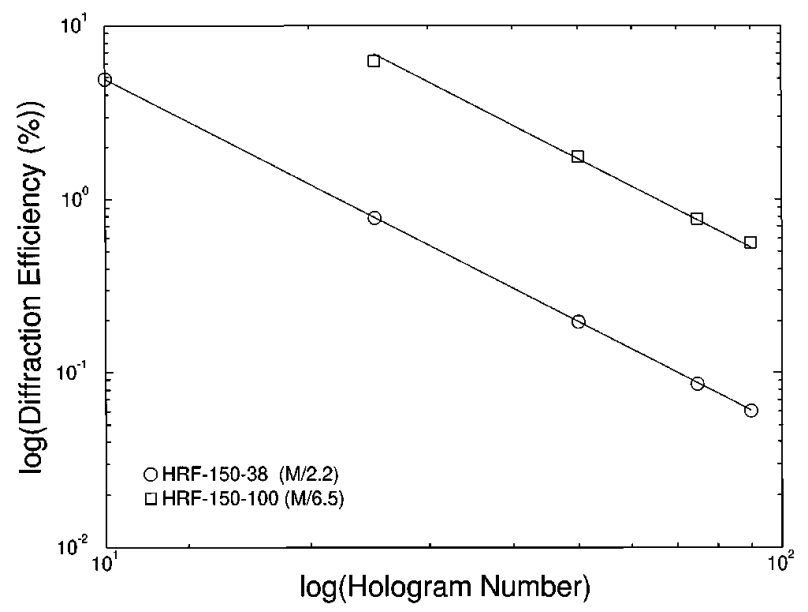

Fig. 8. Diffraction efficiency as a function of the number of holograms stored in Dupont's HRF-150-38, 100- $\mu$ m-thick photopolymer. 
$1 / M^{2}$ theoretical prediction. The $M / \#$ measured from Fig. 8 is $M / 2.2$ and $M / 6.5$ for the 38 - and the 100 - $\mu$ m-thick films, respectively.

If we want to store 1000 holograms in the HRF-150100 photopolymer by using the same setup used to measure the $M / \#\left(\lambda=320 \mathrm{~nm}\right.$ and $\left.\theta_{r}=\theta_{s}=19.2^{\circ}\right)$ then Eq. (9) predicts a diffraction efficiency per hologram of approximately $\eta=4 \times 10^{-5}$. We verified this experimentally by storing 1000 imageplane holograms of a random binary mask by using a combination of peristrophic and angle multiplexing (100 peristrophic holograms at each of the 10 angular locations). The surface density in this case was much lower than $10 \mathrm{bits} / \mu^{2}$ because of the large pixels used. Figure 9 shows the diffraction efficiency as a function of the hologram number for the 1000-hologram experiment (the diffraction efficiency was corrected for the half-on, half-off nature of the binary mask). The result from Fig. 9 was obtained after six iterations through the recording-schedule procedure. The sharp nonuniformities are attributed to stage instability during recording. A reconstruction of one of the 100 stored holograms is shown in Fig. 10. Figure 11 shows the cross section through the same reconstruction. The quality of the reconstructions is excellent.

When the experimentally measured $M / 6.5$ for the HRF-150-100 photopolymer film is used, the diffraction efficiency per hologram for the high-density experiment (32 holograms) should be $\sim 4 \times 10^{-2}$ for each hologram. However, the experimentally measured diffraction efficiency was $\eta=3.5 \times 10^{-3}$. This is attributed to the fact that we used only $30 \%$ of the dynamic range of the material, which effectively reduces $A_{\text {sat }}$ to less than one-third of its value. The diffraction efficiency then drops by the square of this reduction. If we use the actual parameters of our experiment, $N_{p}=590,000, \eta=3.5 \times 10^{-3}, I_{\text {inc }}=1$ $\mathrm{mW}$, and $\lambda_{\text {outside }}=532 \mathrm{~nm}$, then the required integration time becomes approximately $\tau=65 \mu \mathrm{s}$. The expression for the readout rate $R_{\text {out }}$ is simply

$$
R_{\text {out }}=N_{p} / \tau_{\text {out }}
$$

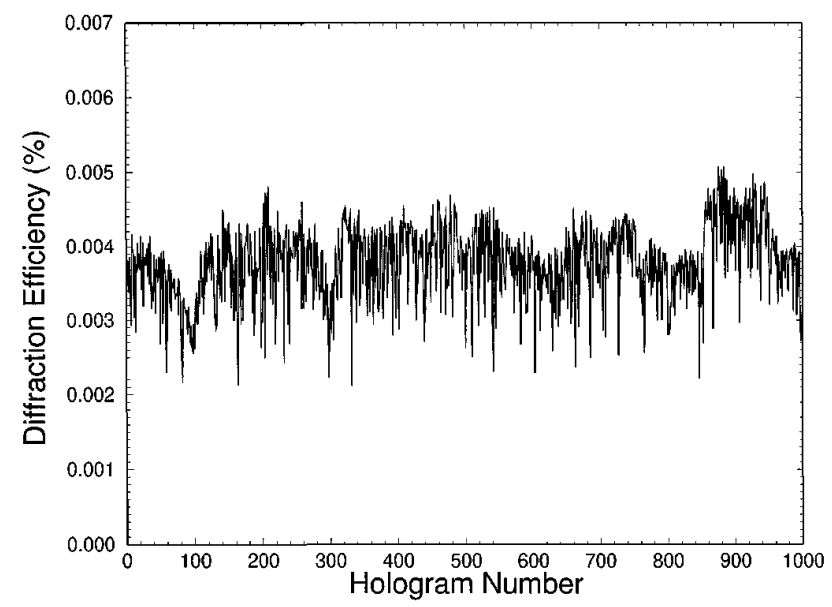

Fig. 9. Diffraction efficiency as a function of the hologram number for the 1000-hologram experiment.

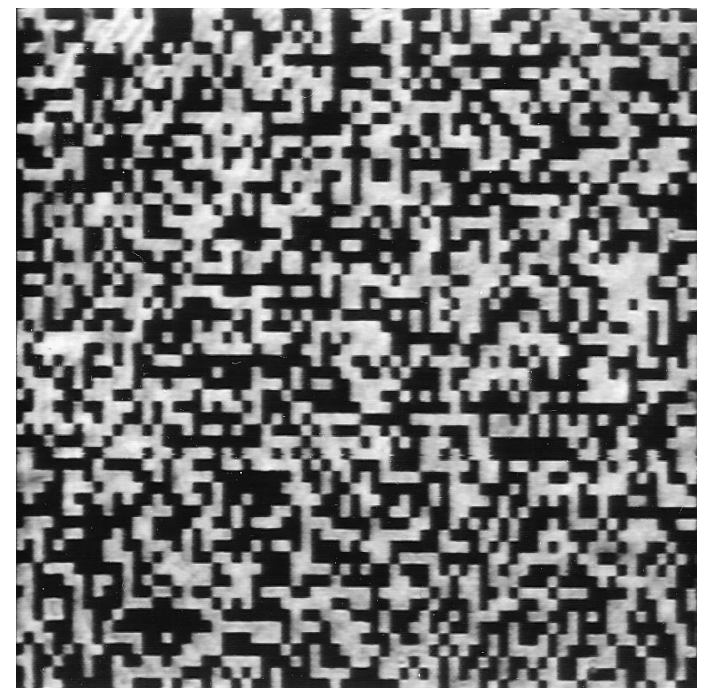

Fig. 10. Reconstruction of one of the 1000 stored holograms.

where $N_{p}$ is the number of pixels per hologram and $\tau_{\text {out }}$ is the time required for reading each hologram. For $\tau=\tau_{\text {out }}=65 \mu \mathrm{s}, R_{\text {out }}=9 \mathrm{Gbits} / \mathrm{s}$ with $N_{p}=$ 590,000 pixels. This readout rate was not actually realized in the experimental setup because the CCD we used cannot read an entire page of data in $65 \mu \mathrm{s}$ and it does not have enough resolution to read all 590,000 pixels. The CCD readout windows of $65 \times$ 65 pixels at a video rate that resulted in the much more modest readout rate of $0.13 \mathrm{Mbits} / \mathrm{s}$ were actually demonstrated in our high-density experiment setup. If a large, parallel-readout CCD detector array is used to read out the entire 590,000 pixels in a minimum frame-transfer rate of 1000 frames/s, then a detector-limited readout rate of greater than $500 \mathrm{Mbits} / \mathrm{s}$ can be achieved.

The motion of the disk introduces an additional constraint in the selection of the integration time on the detector. The motion of the disk causes the reconstructed image on the CCD to move also. If the integration time is too long then the motion of the reconstruction causes smearing. Li and Psaltis ${ }^{17}$

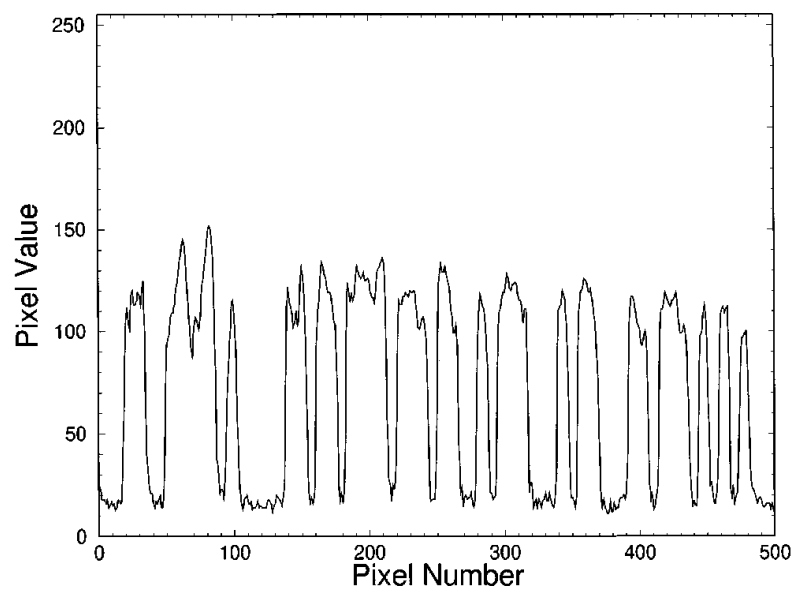

Fig. 11. Cross section of the reconstruction shown in Fig. 10. 
derived the maximum allowable dwell time on the CCD to be $\sim 2.7 \mu \mathrm{s}$, using a disk-rotation rate of 3600 rpm. Therefore, in a continuously spinning disk system, the minimum required integration time cannot be as long as the $65 \mu$ s of the current experimental setup. The integration time can be lowered by a factor of 65 to approximately $1 \mu \mathrm{s}$ by an increase in the laser power or an increase in the diffraction efficiency of the hologram. We can accomplish the same thing by decreasing the number of pixels per page. However, the optical system would have to be redesigned to sustain the same surface density. Also, the recording rate goes down if the number of pixels per page is reduced, but this is not important if the application is a read-only memory. For example, if we use $N_{p}=300,000, \eta=4 \times 10^{-2}$, $I_{\text {inc }}=60 \mathrm{~mW}$, and $\lambda_{\text {outside }}=500 \mathrm{~nm}$, then the required integration time per pixel is only $\sim 50 \mathrm{~ns}$. The laser source could be pulsed for $50 \mathrm{~ns}$ to freeze the reconstruction on the detector and thus avoid smearing while the CCD reads out the detected image. In this case, the read-out rate again becomes limited by the frame-transfer rate of the CCD camera.

\section{Reconstruction Fidelity}

The most challenging aspect of the high-density experiment proved to be the suppression of noise in the system in order to achieve acceptable levels of SNR and probability of error. We reported above the storage of 1000 holograms in the Dupont photopolymer. We were able to retrieve all the bits in the 1000 stored holograms with a very low probability of error (no errors were actually detected in the bits that were tested). Because the high-density experiment requires that only 32 holograms be stored in order to achieve the goal of $10 \mathrm{bits} / \mathrm{\mu m}^{2}$, it may seem simple to demonstrate a low probability error for this experiment. In fact, this was not the case. The way we achieve high density is by increasing the angular bandwidth of the signal and the reference arms. The noise level goes up as the angular bandwidth increases for the majority of noise sources, while the signal level does not. Consequently, the SNR starts to decrease as we attempt to achieve higher surface density, and it becomes necessary to track carefully each of the noise sources and to optimize the system in order to minimize their effect. Through this process we were able to demonstrate storage at $10 \mathrm{bits} / \mu^{2}$ with $\mathrm{SNR}=3.5$ without any errors detected among the bits that were tested. In the remainder of this section we discuss the noise sources in the system and we describe the steps we took to minimize them.

We can divide the noise sources into two categories: system noise and hologram noise. Lens aberrations, SLM imperfections, detector noise, scattering and multiple reflections from lenses and other optical components, laser nonuniformity and flucutations, and SLM-to-CCD pixel misalignment are examples of system noise. The rest of the noise arises from the hologram itself. Specifically, the hologram can introduce cross talk between the recorded holograms, interpixel cross talk, scattering from the recording material, multiple reflections in the medium, nonuniform diffraction efficiency in the recorded holograms, distortions that are due to surface imperfections, blurring that is due to limited spatial resolution of the material, and material shrinkage. We can separately determine the system noise level by simply imaging a page of data presented by the SLM onto the CCD and measuring the SNR of the detected image. After we minimize the system noise, we then record holograms with the system and follow a step-by-step procedure to identify the various sources of hologram noise. We use the following definition of the SNR to make a comparative assessment of system performance:

$$
\mathrm{SNR}=\frac{m_{2}-m_{1}}{\left(\sigma_{1}^{2}+\sigma_{2}^{2}\right)^{1 / 2}},
$$

where $m_{1}$ and $\sigma_{1}$ are the mean and the standard deviations of the intensity of the dark pixels, respectively, and $m_{2}$ and $\sigma_{2}$ are the mean and the standard deviations for the white pixels, respectively. Because the pixel pitch of the SLM is $45 \mu \mathrm{m}$ and the pixel pitch of the CCD array is $10 \mu \mathrm{m}$, each SLM pixel becomes oversampled by the CCD pixels with the unit-magnification imaging system. The CCD detects a window of $65 \times 65$ SLM pixels at a time. The video from the CCD is digitized, and a computer program automatically outlines the SLM pixels in the image. On the average, each SLM pixel is sampled by 20.5 CCD pixels, and the program produces an estimate for the intensity of an SLM pixel by averaging $9.5 \mathrm{CCD}$ pixel values. Therefore, on the average, 11 CCD pixels are discarded as edge pixels when a measurement of a SLM pixel value is made. The data are then processed to compute the mean and the variances so that the SNR in Eq. (11) can be calculated. In addition, a histogram of the data is made, and the data are classified. These measurements provide estimates for the probability of error.

The SNR's obtained for various experimental conditions are plotted in Fig. 12. The first measurement is $\mathrm{SNR}=10$, which is due to system noise only. We obtained this by transmitting the signal beam through the system without any recording material. The histograms of the dark and the white pixels were well separated, and no errors were observed. Next, a glass substrate on which the photopolymer is usually laminated was placed at the recording plane of the system. The SNR dropped to 9 because of the slight aberrations caused by the substrate. When we introduced a piece of UV-cured photopolymer laminated on a glass substrate at the place where the holograms are normally located, the SNR dropped from the upper limit of 9 to $\sim 7$. The major cause of this drop in the SNR is most likely due to internal reflections within the film and the glass substrate and possibly to scattering. The interference fringes 


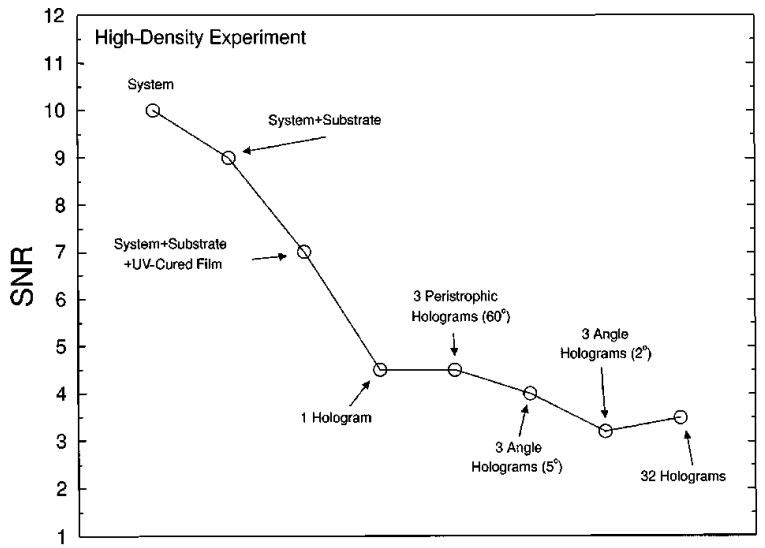

Fig. 12. SNR characterization for the high-density setup.

can be eliminated with antireflection coatings applied to both the glass and the photopolymer.

A SNR of approximately 4.5 was obtained when a single hologram was recorded and its reconstruction was evaluated. The histograms of the dark and the white pixels were still clearly separated, and no errors were observed. This shows that the resolution of the photopolymer is sufficient to record highdensity holograms with clarity. The reason the SNR drops from 7 to 4.5 for one hologram is strongly connected to the internal reflections from the boundaries. As stated above, the signal beam creates an interference pattern (fringes) when it passes through the film and the glass substrate. This is also true for the reference beam when it is brought in to record a hologram. Furthermore, when the reference beam is used to reconstruct the stored holograms, it also creates an interference pattern that can be seen with the naked eye on the film. Therefore the reconstruction suffers from the fringes that are on the reference beam twice. Other effects, such as the fidelity of the recording material, also play roles in degrading the SNR.

The average SNR of three peristropically multiplexed holograms $\left(60^{\circ}\right.$ separation) remains at $\sim 4.5$. This indicates two things: peristrophic multiplexing does not introduce cross talk (as expected), and the superposition of three holograms does not significantly deteriorate the quality of each hologram. The average SNR of three angle-multiplexed holograms $\left(5^{\circ}\right.$ outside separation) drops to $\sim 4$. This is a clear indication of cross-talk noise in the angle holograms. The SNR further drops to $\sim 3.2$ for angle holograms separated by a $2^{\circ}$ outside angle. With a SNR of only 3.2, the histograms of the dark and the white pixels almost overlapped, but no errors were observed. From these measurements, we selected $2.5^{\circ}$ (outside) for the angular separation in the angle-multiplexing portion of the system.

Inherent in the photopolymer material is a shrinkage effect that slightly smears the reconstruction. Furthermore, the shrinkage effect also makes it difficult to Bragg match the entire hologram for readout. For images with low bandwidth, the

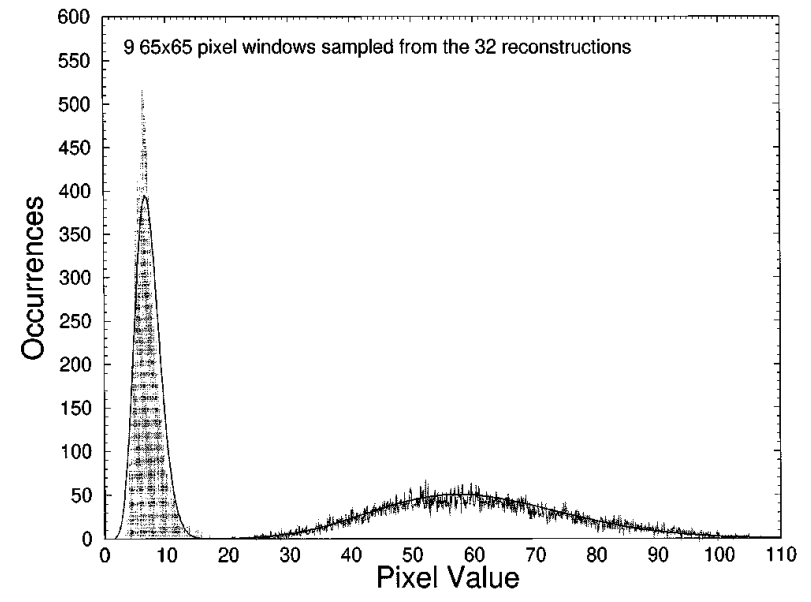

Fig. 13. Combined histogram obtained from nine different sampled windows.

shrinkage effect is barely noticeable in both the hologram quality and the Bragg condition of the entire page. However, in our high-density experiment, the bandwidth of the signal beam alone was $26^{\circ}$ inside the material. This means that, when a hologram is reconstructed, only $\sim 1 / 3$ of the page is Bragg matched at a time. The entire page can be viewed if the angle of the reference beam is changed slightly (or if the stage that does angle multiplexing is rotated). A simple calculation that uses experimental observations reveals that the shrinkage in the HRF-150-100 photopolymer film is $\sim 3 \%$. Not being able to see the entire reconstruction at one time could add substantial complexity to the readout system.

Even with all the noise sources discussed above, we sampled nine different $65 \times 65$ pixel windows from the optimized 32 holograms and no errors were detected. The combined histogram from the nine different sampled windows is shown in Fig. 12. Their average SNR is $\sim 3.5$. The SNR dropped from 4 (obtained when we angle multiplexed three holograms separated by $5^{\circ}$ ) to 3.5 because the cross-talk noise level increased and interpixel gratings started forming because of the 32 exposures. The diffraction efficiency of each hologram was $\eta=3.5 \times 10^{-3}$, whereas the equivalent diffraction efficiency of the light scattered from the polymer when it is illuminated by the reference beam is only $3 \times 10^{-5}$. Therefore scattering is a negligible contributor to the overall noise level. It is likely that there were no errors in any of the 32 stored holograms even though we did not check all the stored bits. We obtained an estimate for the probability of error by fitting a first-order $\chi^{2}$ distribution to the histogram of Fig. 13. The estimated probability of error from this model is approximately $10^{-4}$.

\section{Conclusion}

We experimentally demonstrated a surface density of $10 \mathrm{bits} / \mu^{2}$ in the $100-\mu \mathrm{m}$ Dupont polymer by superimposing 32 holograms, each with a surface 
density of $0.334 \mathrm{bits} / \mu^{2}$. It might be possible to increase the density further by a factor of 2 to 3 by increasing the numerical aperture of the lenses in the system and possibly by reducing the angular separation of the reference beams used for angle multiplexing. However, pressing for substantially higher density in the $100-\mu \mathrm{m}$ film will be difficult. What is really needed is a photopolymer with $1-\mathrm{mm}$ thickness that would yield densities in the range of $100 \mathrm{bits} / \mu^{2}$. The recording rate in the experimental setup was $0.7 \mathrm{Mbits} / \mathrm{s}$, and we extrapolate that $45 \mathrm{Mbits} / \mathrm{s}$ is possible if we use an incident intensity of $128 \mathrm{~mW} / \mathrm{cm}^{2}$. The readout rate of the experimental setup was $0.13 \mathrm{Mbits} / \mathrm{s}$ (limited by the CCD detector), and we project that a readout rate equal to $500 \mathrm{Mbits} / \mathrm{s}$ can be achieved with a CCD that has at least 590,000 pixels and that reads out 1000 frame per second. However, the shrinkage of the polymer remains a serious concern that currently complicates the readout process. No errors were detected in the 38,000 bits that were selected from the 32 reconstructions and evaluated. A theoretical estimate of the expected raw probability of error based on modeling the histogram of the data is approximately $10^{-4}$.

We gratefully acknowledge the U.S. Air Force Office of Scientific Research for sponsoring this research project. We also thank George Barbastathis, Kevin Curtis, and Sidney Li for many helpful discussions.

\section{References}

1. D. Psaltis, "Parallel optical memories," Byte 17(9), 179-182 (1992).

2. H.-Y. S. Li and D. Psaltis, "Three-dimensional holographic disks," Appl. Opt. 33, 3764-3774 (1994).

3. D. L. Staebler, J. J. Amodei, and W. Philips, "Multiple storage of thick holograms in $\mathrm{LiNbO}_{3}$," presented at the Seventh
International Quantum Electronics Conference, Montreal, Quebec, Canada, 1972.

4. F. H. Mok, M. C. Tackitt, and H. M. Stoll, "Storage of 500 high-resolution holograms in a $\mathrm{LiNbO}_{3}$ crystal," Opt. Lett. 16, 504-607 (1991).

5. S. Yin, H. Zhou, F. Zhao, M. Wen, Y. Zang, J. Zhang, and F. T. S. Yu, "Wavelength-multiplexed holographic storage in a sensitive photorefractive crystal using a visible-light," Opt. Commun. 101, 171-176 (1991).

6. G. A. Rakuljic, V. Levya, and A. Yariv, "Optical data storage by using orthogonal wavelength-multiplexed volume holograms," Opt. Lett. 17, 1471-1473 (1992).

7. C. Denz, G. Pauliat, and F. Roosen, "Volume hologram multiplexing using a deterministic phase encoding method," Opt. Commun. 85, 171-176 (1991).

8. Y. Taketomi, J. E. Ford, H. Sasaki, J. Ma, Y. Fainman, and S. H. Lee, "Incremental recording for photorefractive hologram multiplexing," Opt. Lett. 16, 1774-1776 (1991).

9. D. Psaltis, D. Brady, X. G. Gu and S. Lin, "Holography in artificial neural networks," Nature (London) 343, 325-330 (1990).

10. F. H. Mok, "Angle-multiplexed storage of 5000 holograms in lithium niobate," Opt. Lett. 18, 915-917 (1993).

11. K. Curtis, A. Pu, and D. Psaltis, "Method for holographic storage using peristrophic multiplexing," Opt. Lett. 19, 993994 (1994).

12. D. Psaltis, M. Levene, A. Pu, G. Barbastathis, and K. Curtis, "Holographic storage using shift multiplexing," Opt. Lett. 20, 782-784 (1995).

13. Toshiba SD (Super Density), Format tech. spec. (Toshiba, Japan, 1995).

14. K. Curtis and D. Psaltis, "Characterization of the DuPont photopolymer for three-dimensional holographic storage," Appl. Opt. 33, 5396-5399 (1994).

15. G. Zhao and P. Mouroulis, "Diffusion model of hologram formation in dry photopolymer materials," J. Mod. Opt. 41, 1929-1939 (1994).

16. D. Brady and D. Psaltis, "Control of volume holograms," J. Opt. Soc. Am. A 9, 1167-1182 (1992).

17. H.-Y. S. Li and D. Psaltis, "Alignment sensitivity of holographic three-dimensional disk," J. Opt. Soc. Am. A 12, 1902-1912 (1995). 